\title{
Observation and an Explanation of Breakdown of the Quantum Hall Effect
}

M. E. Cage, D. Y. Yu, and G. Marullo Reedtz

National Institute of Standards and Technology, Gaithersburg, MD 20899
We observe a spatially localized breakdown of the nearly dissipationless quantum Hall effect into a set of discrete dissipative states in wide, high-quality GaAs/AlGaAs samples. The phenomenon can be explained by an extension of the quasi-elastic inter-Landau level scattering model of Eaves and Sheard.
Key words: acoustic phonon; breakdown of the dissipationless state; inter-Landau level scattering; population inversion; quantum Hall effect; two-dimensional electron gas.

Accepted: January 29, 1990

\section{Introduction}

The integral quantum Hall resistance [1] $R_{\mathrm{H}}(i)=V_{\mathrm{H}}(i) / I_{x}=h /\left(e^{2} i\right)$ is observed when the longitudinal resistance $R_{x}=V_{x} / I_{x}$ of the two-dimensional electron gas is very small. Here $V_{\mathrm{H}}(i)$ is the Hall voltage of the $i$ th plateau, $i$ is an integer, and $I_{x}$ is the current through the sample. There is a critical current $I_{\mathrm{c}}$ above which $R_{x}$ rapidly increases by several orders of magnitude $[2,3] . R_{x}$ becomes finite as one approaches the critical current. This is referred to as breakdown.

We present results of a breakdown experiment in which sets of discrete $V_{x}$ signals are observed. We propose an explanation based on an extension of the quasi-elastic inter-Landau level scattering (QUILLS) mechanism of Eaves and Sheard [4].

\section{The Experiment}

Our samples [3] were $\mathrm{GaAs} / \mathrm{Al}_{x} \mathrm{Ga}_{1-x}$ As heterostructures grown by molecular beam epitaxy with $x=0.29$. The samples are designated as
$\mathrm{GaAs}(7)$ and $\mathrm{GaAs}(8)$. They have zero magnetic field mobilities of $\sim 100,000 \mathrm{~cm}^{2} /(\mathrm{V} \cdot \mathrm{s})$ at $4.2 \mathrm{~K}$. They exhibit excellent integral quantum Hall effect properties. The inset of figure 1 shows the geometry of the samples. They are $4.6 \mathrm{~mm}$ long and 0.4 $\mathrm{mm}$ wide. Contact was made to the two-dimensional electron gas at points $1,2,3$, and 4, as well as the source $S$ and the drain $D$.

An important feature of the $\mathrm{GaAs}(7)$ sample is that the breakdown occurs somewhere within the longitudinal shaded region between the middle Hall probe pair 3,4 and the outer Hall probe pair 1,2 of figure 1, but not within the transverse region of either Hall probe pair. This crucial fact can be explained by examining figure 1 . The minimum $V_{x}$ signal, $V_{x}^{\min }$, measured between probe pairs 2 and 4 , increases by a factor of $10^{7}$ between $I_{x}=25 \mu \mathrm{A}$ and $I_{x}=370 \mu \mathrm{A}$, but the quantized Hall resistance $R_{\mathrm{H}}$ for the $i=4$ plateau decreases by only one part in $10^{7}$ for probe pair 3,4 at $370 \mu \mathrm{A}$, and by only six parts in $10^{7}$ for probe pair 1,2 . These changes in $R_{H}$ are only about $0.01 \%$ of what was expected from 


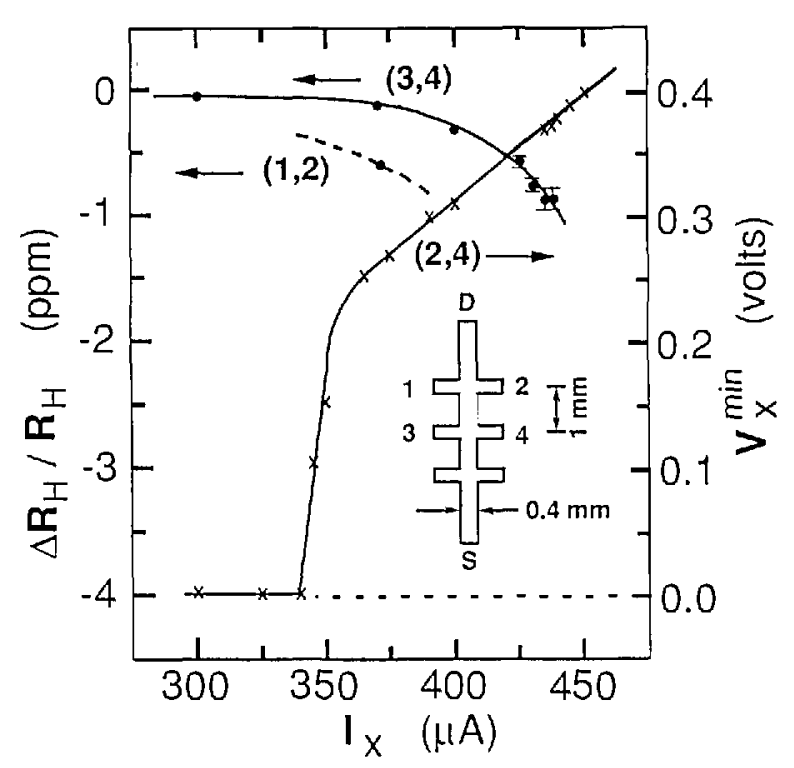

Figure 1. Current dependence of $\Delta R_{\mathrm{H}} / R_{\mathrm{H}}$ and $V_{x}^{\min }$ for the $i=4$ plateau of the $\mathrm{GaAs}(7)$ sample at $1.2 \mathrm{~K}$. The inset shows the sample geometry.

the mixing of $V_{x}$ into $V_{\mathrm{H}}$ due to the known misalignment of the Hall probes. We therefore know the general region where the breakdown occurs, and that the $i=4$ Hall resistance is accurately quantized on both sides of the spacial breakdown region.

The critical current at which $V_{x}^{\text {min }}$ starts to rise abruptly is $I_{\mathrm{c}}=340 \mu \mathrm{A}$. Figure 2 shows a $V_{x}$ vs $B$ curve for $\operatorname{GaAs}(7)$ at $I_{x}=300 \mu \mathrm{A}$, well below that critical current. This curve exhibits reproducible structures. Figure 3, (a) and (b), respectively, show digital oscilloscope displays of the time-dependence and time-averaged distributions of the values of $V_{x}$ obtained at points $\mathrm{A}, \mathrm{B}$, and $\mathrm{C}$ of figure 2 . There are clearly distinct dc voltage levels and switching between levels. Figure 3 clearly shows that the character of these levels changes with magnetic field. Also, $V_{x}$ is in only one state at any given time. It remains in that state until electrical noise or other noise processes induces it to switch to another state. Similar switching between dc voltage levels occurs in the GaAs(8) sample. The $V_{x}$ curve of figure 2 masks this switching by displaying time-averaged values of these dc voltage levels.

We make an initial attempt to interpret our observations by using a modified version of the QUILLS model of Eaves and Sheard [4] because that is a model which seems to provide a satisfactory explanation. Eaves and Sheard used their

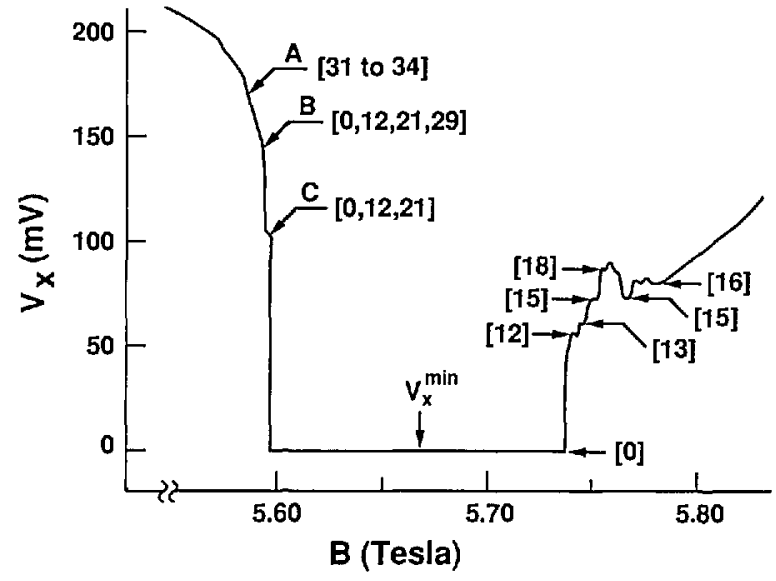

Figure 2. Time-averaged $V_{x}$ signals for the $i=4$ plateau of $\mathrm{GaAs}(7)$ at $1.2 \mathrm{~K}$ and $300 \mu \mathrm{A}$. The number of inter-Landau level transitions $\left(N^{\prime}-N\right)$ is indicated in brackets.

model to interpret the experimental data of Bliek et al. [5] who made breakdown measurements on GaAs/AlGaAs samples in which Hall potential probe sets were placed on either side of a $1 \mu \mathrm{m}$ wide constriction. Their $V_{x}$ vs $B$ curves show features similar to our data, and their Hall voltages were also quantized. Our samples have no geometrical constriction; they are instead $400 \mu \mathrm{m}$ wide. But we propose that the nearly dissipationless conduction channel is very narrow in our samples as the critical current is approached, and that the conduction channel becomes entirely pinched-off and dissipative above the critical current. Our samples therefore may have effective geometries similar to those of Bliek et al. [5] near breakdown. We assume the global current-carrying equipotentials of the percolation model [6], as strongly suggested by experiments which measured the potential distribution across quantum Hall devices [7].

\section{The QUILLS Model}

To explain our data we first extend the QUILLS model of Eaves and Sheard [4] to include transitions between non-contiguous Landau levels. Particles of positive charge $q$ and reduced mass $m^{*}$ (0.068 times the free electron mass in GaAs) move with velocity $v_{x}$ in the positive $x$ direction through a constriction of average length $L_{x}$ and average width $L_{y}$ as shown in figure 4 . The quantities $L_{x}$ and $L_{y}$ can be less than the length $L$ and width $W$ of a geometrical constriction. The average electric field within the constriction and the average mag- 


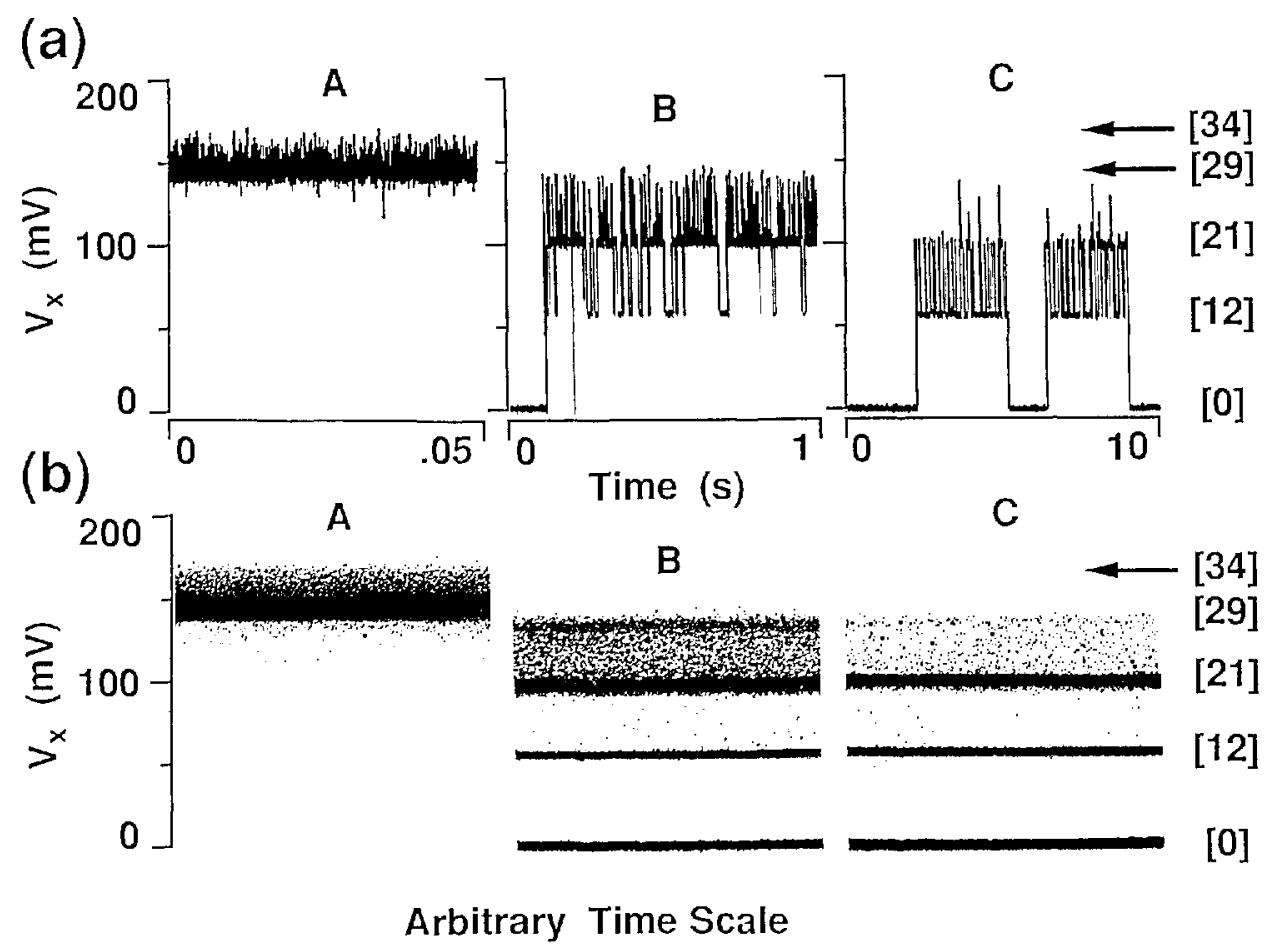

Figure 3. (a) Digital oscilloscope recordings of the time-dependent $V_{x}$ signals at the positions labeled A, $\mathrm{B}$, and $\mathrm{C}$ in figure 2. (b) Digital oscilloscope recordings of the time-averaged distribution of the values of $V_{x}$ at the positions $\mathrm{A}, \mathrm{B}$, and $\mathrm{C}$.

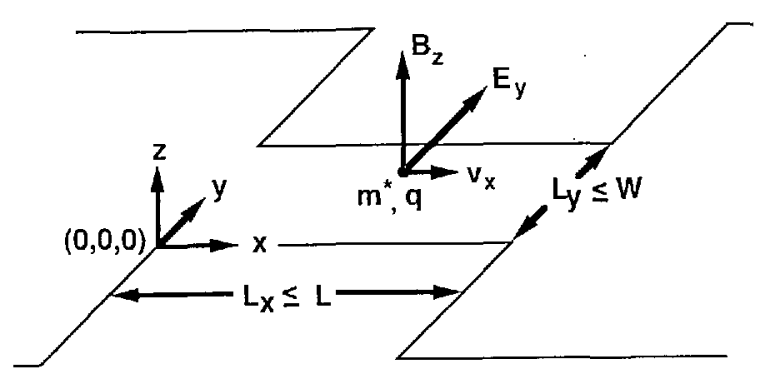

Figure 4. Motion of a charged particle through a constriction. The dynamical length and width $L_{x}$ and $L_{y}$ may be smaller than the physical length and width $L$ and $W$.

netic field is $E_{y}$ and $B_{z}$, respectively. Therefore, $v_{x}=E_{y} / B_{z}$. The Hamiltonian for this system, neglecting spin-splitting and scattering, is $\hat{H}=(1 /$ $\left.2 m^{*}\right)(\hat{p}-q A)^{2}+q V_{y}$ in SI units, where $\hat{p}$ is the momentum operator $-i \hbar \nabla, A$ is the magnetic vector potential and $V_{y}=-y E_{y}$. Using the Landau gauge $A_{x}=-y B_{z}$ and $A_{y}=A_{z}=0$, one obtains the normalized eigenfuctions $\psi$ and energy eigenvalues $\mathscr{E}$ to Schodinger's equation $\hat{H} \psi=\mathscr{E} \psi$ :

$$
\begin{aligned}
& \psi_{N, k_{x}}(x, \xi)=\frac{1}{\left(L_{x}\right)^{1 / 2}} \mathrm{e}^{i k_{x} x} \frac{1}{\left(2^{N} N !\right)^{1 / 2}} \frac{1}{(\pi)^{1 / 4}} \mathrm{e}^{-\xi^{2} / 2} H_{N}(\xi) \\
& \psi_{N, k_{x}}(x, \xi)=\frac{1}{\left(L_{x}\right)^{1 / 2}} \mathrm{e}^{i k_{x} x} \Phi_{N}(\xi) \\
& \mathscr{E}_{N}\left(\mathrm{y}_{0}\right)=\left(N+\frac{1}{2}\right) \hbar \omega_{\mathrm{c}}-q y_{0} E_{y}+\frac{1}{2} m * v_{x}^{2},
\end{aligned}
$$

where $N$ is the Landau level number, $\omega_{\mathrm{c}}=q B_{z} / m^{*}$ is the cyclotron angular frequency, $\ell_{B}=\left(\hbar / q B_{z}\right)^{1 / 2}$ is the magnetic length, $y_{0}=\left(v_{x} / \omega_{\mathrm{c}}-\ell_{B}^{2} k_{x}\right)$ is the $y$ coordinate of the center of motion of each cyclotron orbital, $k_{x}=2 \pi N_{k} / L_{x}$ is the $x$ component of the wavevector of a cyclotron orbital state $\left(N, N_{k}\right), N_{k}$ is the integer quantum number of the wavevector of that state, $\xi=\left(y-y_{0}\right) / \ell_{B}$, and $H_{N}(\xi)$ is a Hermite polynomial.

Figure 5 shows the energy eigenvalues for Landau level $N$ as a function of $y$. The slope of the lines is $-q E_{y}$. The eigenvalues are equally spaced, with separation $\Delta y_{0}=2 \pi \ell_{B}^{2} / L_{x}$. The maximum number of allowed extended states $\left(N, N_{k}\right)$ is $\left(q B_{z}\right)$ h) $L_{x} L_{y}$ for level $N$. The total number of allowed extended states per unit area is $n_{\mathrm{s}}=i\left(q B_{z} / h\right)$. 


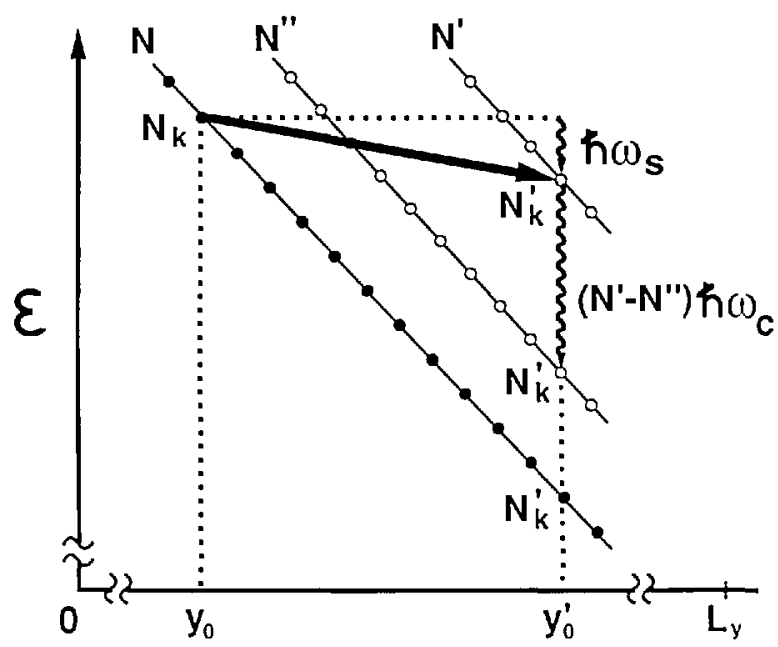

Figure 5. Total energy eigenvalues $\mathscr{E}_{N}$ as a function of position $y$ across the constriction for each Landau level $N$. Every eigenvalue of level $N$ has a unique quantum number $N_{k}$. The figure shows a QUILLS transition from level $N$ to $N^{\prime}$ and an associated acoustic phonon. The decay, either directly down to level $N$ or through an intermediate level $N^{\prime \prime}$, and its associated optical phonon, are also shown.

If $L_{y}$ becomes small enough and $E_{y}$ large enough then it may be possible for a particle to make a transition from state $\left(N, N_{k}\right)$ to an empty state $\left(N^{\prime}, N_{k}^{\prime}\right)$ at a lower total energy, as shown in figure 5. The particle moves across the sample in this model from position $y_{0}$ to $y_{0}^{\prime}$. Energy and momentum must be conserved in the transition. Therefore, an acoustic phonon of wavevector

$$
K_{x}=\frac{2 \pi}{L_{x}}\left(N_{k}-N_{k}^{\prime}\right)=\frac{\omega_{c}}{\left(v_{x}-v_{s}\right)}\left(N^{\prime}-N\right)
$$

and energy $\mathscr{C}_{\text {phonon }}=\hbar \omega_{\mathrm{s}}=\hbar v_{\mathrm{s}} K_{x}$ is emitted in the $x$ direction, where $v_{\mathrm{s}}$ is the velocity of sound in that medium $\left(\sim 2.47 \times 10^{3} \mathrm{~m} / \mathrm{s}\right.$ in GaAs[8]).

Only transitions in which the ratio $\left(y_{0}^{\prime}-y_{0}\right) L_{x} /$ $\left(2 \pi \ell_{B}^{2}\right)$ is an integer number $\left(N_{k}-N_{k}^{\prime}\right)$ are allowed. One can obtain an equation for $\left(N_{k}-N_{k}^{\prime}\right)$ by estimating the value of $\left(y_{0}^{\prime}-y_{0}\right)$. This is accomplished by noting that the spatial extent of $\psi_{N, k_{x}}(x, \xi)$ or $\Phi_{N}(\xi)$ is approximated well by the amplitude of motion of a classical harmonic oscillator: $A_{N}=\ell_{B} \sqrt{2 N+1}$. Transitions can commence when the wavefunctions just begin to significantly overlap:

$$
\left(y_{0}^{\prime}-y_{0}\right) \approx\left(A_{N}+A_{N}^{\prime}\right)=\ell_{B} \xi_{c \ell}^{N, N}
$$

where $\xi_{c, e^{\prime}}^{N, N^{\prime}}=\sqrt{2 N+1}+\sqrt{2 N^{\prime}+1}$. Figure 6 shows such an overlap between $\Phi_{0}(\xi)$ and $\Phi_{12}(\xi)$. Thus

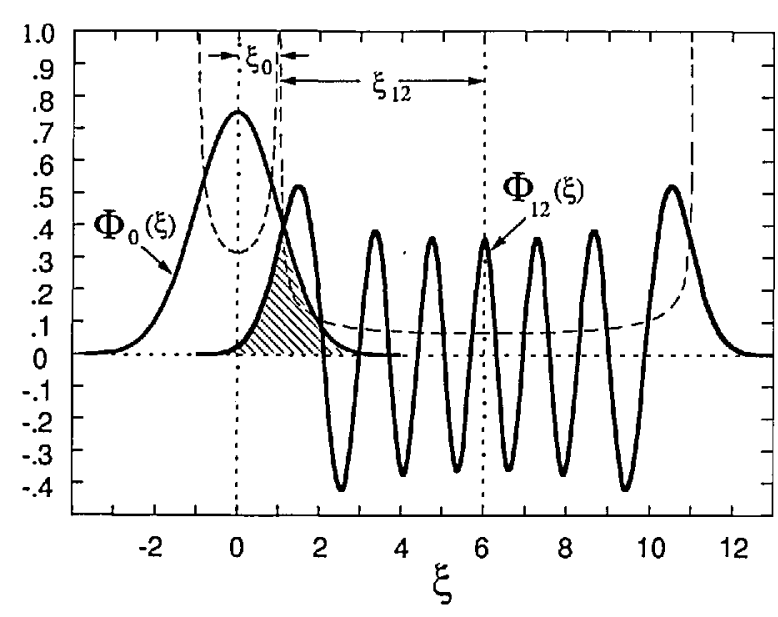

Figure 6. The wavefunctions $\Phi_{0}(\xi)$ and $\Phi_{12}(\xi)$, shown overlapping at the classical harmonic oscillator separations $\xi_{0}$ and $\xi_{12}$. The dashed lines represent the classical harmonic oscillator probability distributions.

$$
\left(N_{k}-N_{k}^{\prime}\right) \approx \frac{L_{x}}{2 \pi \ell_{B}} \xi_{c \ell}^{N, N}
$$

There is another condition for the transition: $\left(N^{\prime}-N\right)$ must also be an integer. From conservation of energy, the electric field $E_{y}=V_{\mathrm{H}} / L_{y}$ is

$$
E_{y}=\left[\left(N^{\prime}-N\right) \hbar \omega_{\mathrm{c}}+\hbar \omega_{\mathrm{s}}\right] / q\left(y_{0}^{\prime}-y_{0}\right) .
$$

The simultaneous integer conditions $\left(N_{k}-N_{k}^{\prime}\right)$ and $\left(N^{\prime}-N\right)$, given by eqs (3) and (4), occur only at particular values of the current and magnetic field. There may be many intervening Landau levels between $N$ and $N^{\prime}$ in which these conditions are not satisfied. Notice that the filled states of Landau level $N$ are at a higher total energy than those of the unoccupied $N^{\prime}$ level. The large Hall electric field $E_{y}$ has induced a population inversion for QUILLS transitions.

After the particles make the $N$ to $N^{\prime}$ QUILLS transitions they then decay, by optical phonon emission [9], back down to the original Landau level $N$, either directly or by cascading through intermediate levels $N^{\prime \prime}$, as shown in figure 5. The optical phonon transitions probably occur just outside of the constriction. One can calculate the voltage signal $V_{x}$ from the electrical power dissipated, $P=I_{x}^{2} R_{x}=I_{x} V_{x}$, when the particles return to the lowest Landau level during the average time taken to traverse the constriction:

$$
V_{x}=\frac{1}{q} \frac{n^{\prime}}{n_{\mathrm{s}}}\left[\left(N^{\prime}-N\right) \hbar \omega_{\mathrm{c}}+\hbar \omega_{\mathrm{s}}\right],
$$


where $I_{x}=q n_{s} v_{x} L_{y}$, and $n^{\prime} / n_{s}$ is the fraction of conducting particles that make the transition. We assume both spin states can undergo transitions. Therefore $N$ is 0 and $n^{\prime} / n_{\mathrm{s}} \leqslant 1$ for the $i=2$ plateau. $N$ is either 0 or 1 and $n^{\prime} / n_{s} \leqslant 1 / 2$ for the $i=4$ plateau. If all the electrons make the transition, then $n^{\prime} / n_{\mathrm{s}}$ is 1 and $1 / 2$ for the $i=2$ and 4 plateaus.

\section{Analysis of Our Data}

We next apply this QUILLS model to our $V_{x}$ vs $B$ data at $300 \mu \mathrm{A}$. Equation (5) is used first to obtain the values of $\left(N^{\prime}-N\right)$ by ignoring the small term $\hbar \omega_{s}$. The electric field $E_{y}$ is then calculated from eqs (4) and (2) for specific values of $V_{x}$ and $B_{z}$. $E_{y}$ ranges between $1.6-3.9 \times 10^{6} \mathrm{~V} / \mathrm{m}$ across the quantized Hall resistance plateau. The constriction width $L_{y}=V_{\mathrm{H}} / E_{y}=I_{x} R_{\mathrm{H}} / E_{y}$ varies between $0.5-1.2$ $\mu \mathrm{m}$ across the plateau, and is narrower on either side of the $V_{x} \approx 0$ region. The range of $v_{x}=E_{y} / B_{z}$ is $2.8-7.0 \times 10^{5} \mathrm{~m} / \mathrm{s}$ across the plateau, and $v_{x}$ is $\sim 200 v_{s}$. Also, $\hbar \omega_{\mathrm{s}}=\hbar v_{\mathrm{s}} K_{x}$, calculated from eq (1), is $\sim 0.5 \%$ of $\left(N^{\prime}-N\right) \hbar \omega_{c}$. The dissipation of the QUILLS transitions is therefore very small indeed. The current density $J_{x}=I_{x} / L_{y}=E_{y} /\left(h / 4 q^{2}\right)$ varies between $240-600 \mathrm{~A} / \mathrm{m}$. This is an astonishingly large number compared with that found in [5], but the current has a better opportunity of finding a dissipationless path in wide high-quality samples. Also, $q V_{\mathrm{H}} \approx 200 \hbar \omega_{c}$. This is very large compared with our deduced $\left(N^{\prime}-N\right) \hbar \omega_{c}$ values, and is in an entirely different regime than that of Kirtley et al. [10] who found small integer values of $q V_{\mathrm{H}} / \hbar \omega_{\mathrm{c}}$. $\left(N_{k}-N_{k}^{\prime}\right)$ is the only quantity that can not be deduced from the data because the length of the constriction $L_{x}$ is unknown. If $L_{x}$ is assumed to be comparable to the width, i.e., about $1 \mu \mathrm{m}$, then $\left(N_{k}-N_{k}^{\prime}\right) \approx 90$ from eq (3). Finally, eq (5) predicts that the quantity $\left(n_{\mathrm{s}} / n^{\prime}\right)\left(m^{*} / \hbar\right) V_{x} /\left[\left(N^{\prime}-N\right) B_{z}\right]$ should be nearly equal to 1 . This quantity is within $3 \%$ of 1 for the $\left(N^{\prime}-N\right)$ transitions shown in figure 2, and is within our experimental accuracy. All of the electrons in Landau level $N$ apparently make the transition to level $N^{\prime}$.

Multiple values of $V_{x}$ sometimes occur at certain values of $B_{z}$, as for example at points $\mathrm{B}$ and $\mathrm{C}$ of figures 2 and 3 . In such cases the above equations yield a different value of $L_{y}$ for each value of $V_{x}$. A more realistic approach in those situations is to use the smallest value of $L_{y}$ obtained from the largest value of $V_{x}$ for that magnetic field. The values of $L_{y}$ and $E_{y}$ are then constant for all QUILLS transitions observed at a given $B_{z}$ and $I_{x}$. With these as- sumptions, the values of $\xi^{N, N}$ for the smaller $V_{x}$ transitions are then less than the classical values $\sqrt{2 N+1}+\sqrt{2 N^{\prime}+1}$. This presents no problem because the overlap integral between $\Phi_{N}(\xi)$ and $\Phi_{N}\left(\xi^{\prime}\right)$ becomes somewhat larger inside of the classical value and then falls off slowly over a wide region as $\xi^{N, N}$ is reduced.

The features labeled with the $\left(N^{\prime}-N\right)$ values 12 , $13,15,16$, and 18 on the right hand side of the $V_{x}$ curve of figure 2 have very stable single-valued signals. This is not always the case; for example, we see switching about the $\left(N^{\prime}-N\right)=12$ transition at position $\mathrm{C}$ and about the $\left(N^{\prime}-N\right)=21$ transition at position $\mathrm{B}$. We believe that the switching is noiseinduced.

We clearly observe discrete levels of $V_{x}$ in figure 3. It is difficult to understand why the overlap of the rather broad wavefunctions and the integer requirements of the apparently large values of $\left(N^{\prime}-N\right)$ and $\left(N_{k}-N_{k}^{\prime}\right)$ would by themselves lead to preferential $N$ to $N^{\prime}$ inter-Landau level transitions. An additional, unaccounted selection mechanism may be present.

One has to be careful about the definition of critical current. For example, in our experiment $V_{x}$ is large and there appears to always be dissipation at points $\mathrm{B}$ and $\mathrm{C}$ in figure 2. However, when looking at the discrete voltage states of those points in figure 3 we see that for one of those states $\left(V_{x} \approx 0\right)$ the dissipation is negligible. Therefore the critical current has not yet been exceeded.

\section{Discussion}

Our interpretation of the QUILLS model for breakdown is consistent with the experiment of Bliek et al. [5], who made physical constrictions of order $1 \mu \mathrm{m}$ width and $10 \mu \mathrm{m}$ length. They observed step-like structures in their $V_{x}$ vs $B$ curves and empirically found equations for the quantized values of $R_{x}$ at those steps and for the magnetic field values at which the structures occur. The empirical number $n_{L}=L_{x}\left(2 q B_{z} / h\right)^{1 / 2}$ of Bliek et al. [5] corresponds to $n_{L}=\left(N_{k}-N_{k}^{\prime}\right)(4 \pi)^{1 / 2} / \xi_{c \ell}^{N, N}$ in our formulation, whereas Eaves and Sheard [4] predicted that $n_{L}=\left(N_{k}-N_{k}^{\prime}\right)$. The equation $R_{x}=n_{R}\left(n_{L} /\right.$ $\left.n_{O}\right)\left(h / 2 \mathrm{e}^{2}\right)$ of [5] corresponds to our interpretation of QUILLS if $n_{R}=\left(n^{\prime} / n_{\mathrm{s}}\right) \xi_{c \ell^{\prime}}^{N^{\prime}} /(\pi)^{1 / 2}$ and $n_{0}=n_{\mathrm{s}} L_{x} L_{y}$. Eaves and Sheard [4] predict that $n_{R}$ is an integer number. Neither $n_{L}$ nor $n_{R}$ are integers in our formulation.

In our interpretation of the data of Bliek et al. [5] the values of $\left(N^{\prime}-N\right)$ vary between 3 and 13 for 
their $i=2$ plateau assuming, as they did, that their $V_{x}$ signal is not a time average of several Landau level transitions. They obtained values of $n_{R}$ between 2 and 19. We find that the effective constriction of their sample $L_{y}$ varies between about $0.2-0.5$ $\mu \mathrm{m}$, significantly less than the $1 \mu \mathrm{m}$ physical width. If $L_{x}$ equals the physical $10.3 \mu \mathrm{m}$ length $L$, then $\left(N_{k}-N_{k}^{\prime}\right)$ ranges between about $650-990 . L_{x}$ is probably much less than $L$, yielding smaller values of $\left(N_{k}-N_{k}^{\prime}\right)$. Bliek et al. [5] found that $n_{L}$ varied between 528 and 607 .

Sachrajda et al. [11] also used samples with a narrow constriction. They observed structures having the same triangular behavior as that of the critical current versus magnetic field plot of Bliek et al. [12]. They believed that this behavior did not agree with the QUILLS model. But we find from our data that the conduction channel narrows on either side of $V_{x} \approx 0$, and because less current is required in a narrower channel to obtain the same electric field, our results show that the QUILLS model is consistent with that behavior.

It has been proposed $[13,14]$ that breakdown is due to emission of phonons in a manner analogous to the Cherenkov effect if $v_{x}>v_{\mathrm{s}}$. In the QUILLS interpretation of our data $v_{x} \approx 200 v_{s}$ and the Cherenkov angle $\theta_{\mathrm{c}}=\cos ^{-1}\left(v_{\mathrm{s}} / v_{\mathrm{c}}\right)$ varies between $89.5^{\circ}$ and $89.8^{\circ}$. The dissipative voltage $V_{x}$ can be very small even when the velocity $v_{x}$ is apparently quite large. The experiment of Bliek et al. [5] provides excellent proof that this may indeed be the case. Figure 1 of their paper shows that $V_{x}$ has structures on the sides of the $i=2$ plateau and goes to zero in the center of the plateau for $I_{x}=45 \mu \mathrm{A}$, $L_{y} \approx 1 \mu \mathrm{m}$, and $B_{z} \approx 6.4 \mathrm{~T}$. Therefore, $v_{x}=E_{y} /$ $B_{z}=\left(I_{x} R_{\mathrm{H}}\right) /\left(L_{y} B_{z}\right) \approx 36.7 v_{\mathrm{s}}$, and yet $V_{x}$ still goes to zero.

We have considered the acoustic phonon emission to be collimated in the $x$ direction. However, the electron gas has a probability distribution in the $z$ direction that extends over an interval $\Delta z$ which is about $50 \AA$ in GaAs/AlGaAs. Therefore the acoustic phonons may have $K_{z} \sim 1 / \Delta z$ components which are about $35 \%$ of the magnitude of the $K_{x}$ component in our experiment for the $\left(N^{\prime}-N\right)=12$ transition. Phonon emission in the $x-z$ plane would not have a significant effect on the results of this paper, but it might preclude the possibility of spontaneously emitted phonons stimulating the other phonons to make population-inverted QUILLS transitions in a manner analogous to photon emission of lasers.

\section{Conclusions}

We have observed spatially localized breakdown of the nearly dissipationless quantum Hall effect into a discrete set of dissipative states, and have interpreted these observations in terms of the QUILLS model. Theoretical analyses of QUILLS transition rates, similar to [13], and optical phonon decay rates would be very useful in determining the validity of QUILLS. Direct observation of the acoustic phonons would confirm the QUILLS process. There are other interesting features that could be studied: the decay process; the quantum heater property of $R_{x}$; and the fact that two crossed resistances, $R_{\mathrm{H}}$ and $R_{x}$, are both quantized. We suggest that QUILLS transitions may be the dominant breakdown mechanism in high-quality integral quantum Hall effect samples, and that the discrete $V_{x}$ signals are indicators of those transitions.

\section{Acknowledgments}

The authors thank A. C. Gossard who made the MBE-grown GaAs/AlGaAs heterostructures and D. C. Tsui who did the photolithography and ohmic contacting. The authors also thank C. T. Van Degrift, S. M. Girvin, E. R. Williams, R. S. Davis, B. N. Taylor, A. F. Clark, K. C. Lee, and R. E. Elmquist for their discussions and comments. This work was partially supported by the Calibration Coordination Group of the Department of Defense, the Naval Strategic System Programs Office, and Sandia National Laboratories.

About the authors: Marvin E. Cage is a physicist in the Electricity Division of the NIST Center for Electronics and Electrical Engineering. Dingyi $Y u$ is a physicist from the Shanghai University of Science and Technology, Shanghai, China and is a guest researcher at NIST. Giancarlo Marullo Reedtz is a physicist at the Galileo Ferraris National Electrotechnical Institute, Turin, Italy and was a guest researcher at NIST.

\section{References}

[1] von Klitzing, K., Dorda, G., and Pepper, M., Phys. Rev. Lett. 45, 494 (1980).

[2] Ebert, G., von Klitzing, K., Ploog, K., and Weimann, G., J. Phys. C 16, 5441 (1983). 
[3] Cage, M. E., Dziuba, R. F., Field, B. F., Williams, E. R., Girvin, S. M., Gossard, A. C., Tsui, D. C., and Wagner, R. J., Phys. Rev. Lett. 51, 1374 (1983).

[4] Eaves, L., and Sheard, F. W., Semicond. Sci. Technol. 1, 346, (1986).

[5] Bliek, L., Hein, G., Kose, V., Niemeyer, J., Weimann, G., and Schlapp, W., Proc. Intern. Conf. on the Application of High Magnetic Fields in Semiconductor Physics, ed. by Landwehr, G., Springer-Verlag, Berlin, 71, (1987), p. 113.

[6] Kazarinov, R. F., and Luryi, S., Phys. Rev. B 25, 7626 (1982).

[7] Ebert, G., von Klitzing, K., and Weimann, G., J. Phys. C 18, L257 (1985); Zheng, H. Z., Tsui, D. C., and Chang, A. M. Phys. Rev. B 32, 5505 (1985).

[8] McSkimin, H. J., Jayaraman, A., and Andreatch, P., J. Appl. Phys. 38, 2362 (1967).

[9] Slater, G. W., and Tremblay, A.-M. S. Phys. Rev. B 29, 2289 (1984).

[10] Kirtley, J. R., Schlesinger, Z., Theis, T. N., Milliken, F. P., Wright, S. L., and Pallmateer, L. F., Phys. Rev. B 34, 5414 (1986).

[11] Sachrajda, A. S., Landheer, D., Boulet, R., and Moore, T., Phys. Rev. B 39, 10460 (1989).

[12] Bliek, L., Braun, E., Hein, G., Kose, V., Niemeyer, J., Weimann, G., and Schlapp, W., Semicond. Sci. Technol. 1, 110 (1986).

[13] Heinonen, O., Taylor, P. L., and Girvin, S. M., Phys. Rev. B 30, 3016 (1984).

[14] Streda, P., and von Klitzing, K., J. Phys. B 59, 379 (1985). 